\title{
Characterization of Luminance Probe for Accurate Contrast Measurements in Medical Displays
}

Edward F. Kelley

Aldo Badano 


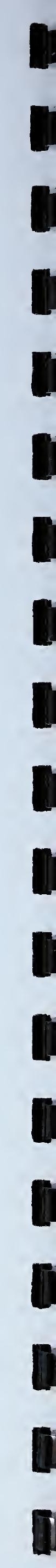




\title{
Characterization of Luminance Probe for Accurate Contrast Measurements in Medical Displays
}

\author{
Edward F. Kelley \\ Electricity Division \\ Electronics and Electrical Engineering Laboratory \\ National Institute of Standards and Technology \\ Gaithersburg, MD 20899-8114 \\ Aldo Badano \\ Center for Devices and Radiological Health \\ Food and Drug Administration \\ Department of Health and Human Services \\ Rockville, MD 20857
}

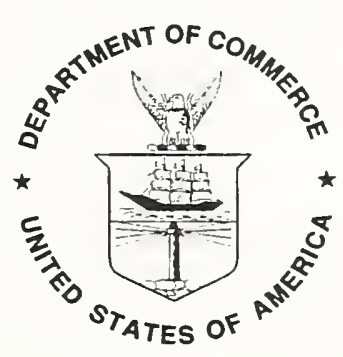

U.S. DEPARTMENT OF COMMERCE

Donald L. Evans, Secretary

TECHNOLOGY ADMINISTRATION

Phillip J. Bond, Under Secretary of Commerce for Technology NATIONAL INSTITUTE OF STANDARDS AND TECHNOLOGY Arden L. Bement, Jr., Director 


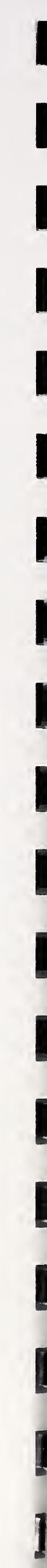




\section{Characterization of Luminance Probe for Accurate Contrast Measurements in Medical Displays}

\section{ABSTRACT}

Quantifying the luminance range and image quality of a diagnostic $\mathrm{x}$-ray image presented in medical electronic displays for radiology workstations is difficult because of the extremely high contrasts involved. Methods are presented here that permit the evaluation of the performance of a detector probe used in an attempt to accurately measure these high contrast images. The contamination of the measured displayed luminance arising from the reflections back off the detector apparatus are compared with the internal veiling-glare corruption that occurs from scattering within the detector probe. The veiling-glare from scattering is seen to be the major contribution to errors in luminance measurements using a simple detector within the probe. Suggestions for improvements are presented for future work.

\section{KEY WORDS}

flat panel display, FPD, narrow frustum SLET, probe, medical display, stray-light elimination tube (SLET), radiological display

\section{INTRODUCTION}

Non-trivial images are those that exhibit a substantial range of contrast such as diagnostic images acquired using medical digital imaging systems. These images can have a 10-bit grayscale depth and up to 5 million pixels. A specialist that makes a medical decision as to the presence of abnormalities performs the interpretation of these images off electronic displays. The accurate measurement of the luminance in such complex images can be difficult. Two problems can arise: (1) The bright regions can produce veiling glare within the detector thereby corrupting the measurement of dark regions, and (2) the detector apparatus can reflect light back onto the display device and cause a contamination of the observed luminance. (Note that the veiling glare referred to in this report is the stray light generated within the detector and does not refer to stray light in the light source whether it be film, a display, or other source of light.) We will limit ourselves to the discussion of luminance measurements in this report. However, the same kind of corruption can occur in making color measurements of detailed images whenever the local color contrast is high in the vicinity of the measurement. It should be noted that veilingglare corruption of the measurement is not confined to high-contrast images. Substantial errors can be made in measuring uniform fields of color whenever the instrument exhibits a sensitivity to the size of the area exposed to the detector. ${ }^{1,2}$

Methods to accurately measure high-contrast images-such as a small black area on a white field-using a gloss black frustum (a cone with its tip cut off) have been employed for display measurements in general, and the advantages over flat masks have been noted. ${ }^{3}$ Frusta (or frustums) have also been employed in a tubular structure to make measurements on projection displays-the device is termed a stray-light-elimination tube (SLET). ${ }^{4}$ An extension of this concept using a small frustum with a much narrower apex angle has been introduced for the purposes of measuring high-contrast medical displays. ${ }^{5}$ The purpose of this report is to describe a method to characterize such narrow-frustum SLETs using either flat or frusta interior apertures. For simplicity, we will refer to such narrow-frustum SLETs as probes. Such probes can be used with photopic photodiode detectors or with array detectors with lenses. ${ }^{6}$ The characterization amounts to determining how much veiling glare is generated within the probe and how much contamination to the measurement is introduced from reflections off of the probe back onto the display device being measured. We will demonstrate that for the probe construction employed here, the veiling glare generated within the probe dramatically dominates any contamination contribution arising from reflections owing to the probe's presence in near proximity to the display surface.

The purpose of the measurement results reported here are to characterize phenomena related to the use of these probes. Such a characterization does not depend upon an absolute calibration of the instrumentation. As such, all photometric results reported here are estimated to have a relative expanded uncertainty with a coverage factor of two of $10 \%$. The reason for the somewhat large uncertainty arises not from the precision capabilities of the apparatus, but the fundamental calibration of the instruments used to calibrate this apparatus.

\section{APPARATUS}

Figure 1 shows the overall view of the apparatus and identifies its major parts. The probe has a photopic photodiode that produces a current $J_{\mathrm{p}}$ that is intended to represent the luminance $L_{\mathrm{p}}$ measured by the probe. The presence of the probe reflects light back upon the target being measured. Here the target consists of an opaque annulus surrounding the illuminance head. That reflected light is measured as an illuminance $E_{\mathrm{p}}$ by the fiber-optic illuminance head. The-illumination is provided by an integrating box light source that is monitored by a photopic photodiode producing current $J_{\mathrm{s}}$. The fiber-optic cable is painted white within the box and exits the box to the detection apparatus. The output of the fiber-optic cable is input to a small 


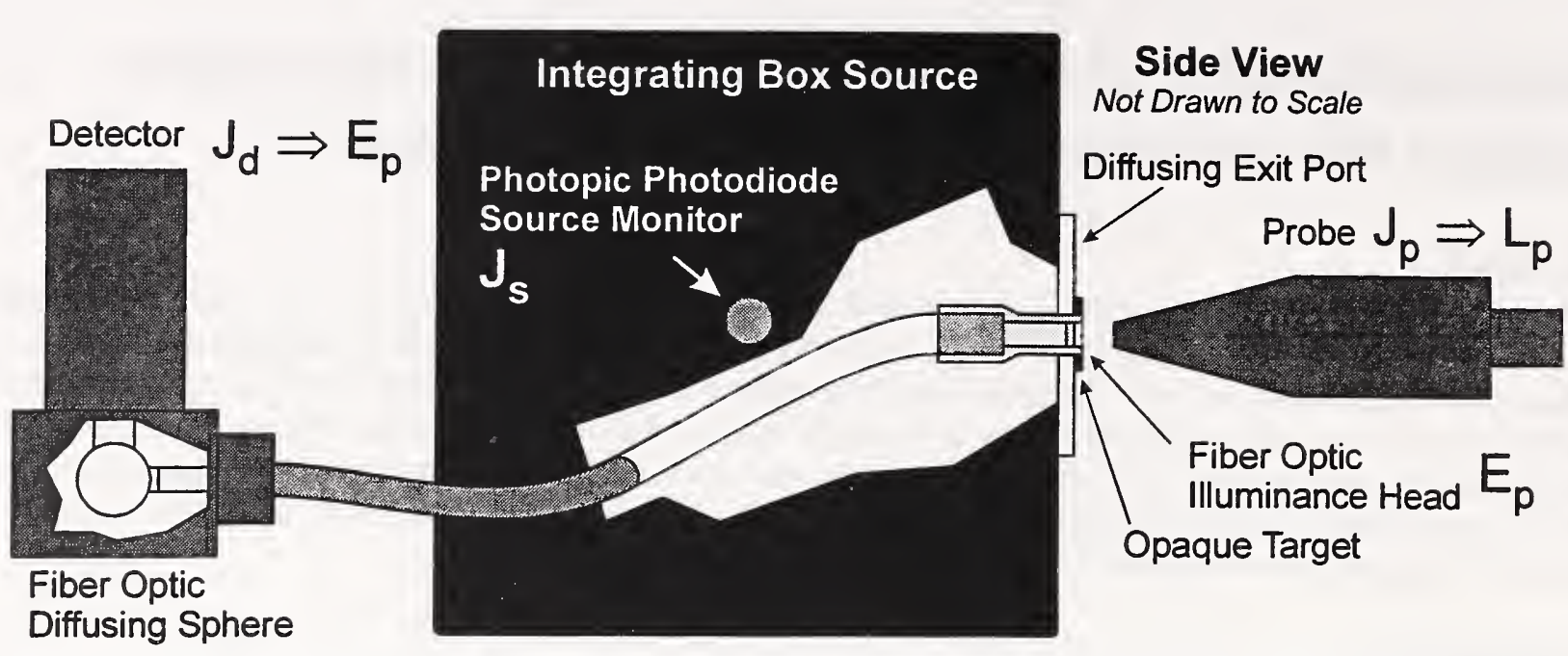

Fig. 1. Overview of apparatus showing the measurement probe, the integrating box source, and the detector based upon a fiber-optic cable. Not drawn to scale.

integrating sphere--the fiber-optic diffusing sphere-the light within which is monitored by a photopic detector producing a photocurrent $J_{\mathrm{d}}$ proportional to illuminance $E_{\mathrm{p}}$. The box source is placed upon a motorized $x-y$-z-positioner that can be moved independently of the probe. We will describe the details of the apparatus in sections: first the source, then the illuminance detector, and finally the probe.

\section{INTEGRATING BOX SOURCE}

CONSTRUCTION: Some of the details of the integrating box source are shown in Fig. 2. The box is made from closed-cell polystyrene foam. ${ }^{8}$ The exterior of the box is painted matte black. Fluorescent bulbs are used as lamps that are supplied with ac power from a saturated ferroresonant transformer to regulate the power supplied to the lamps. The lamps are

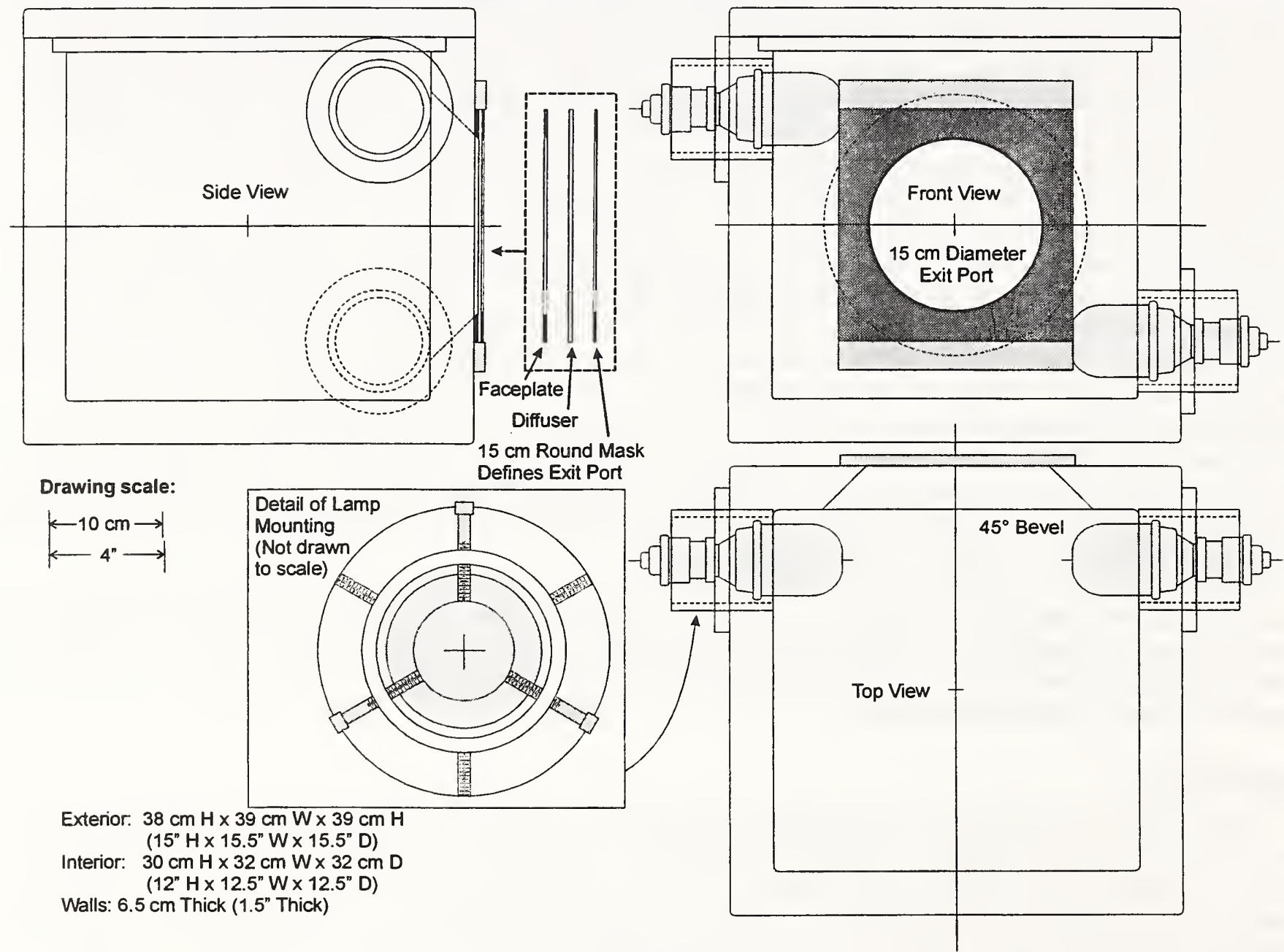

Fig. 2. Polystyrene box integrating source with fluorescent lamps. The exterior of the box is painted matte black. The fiber-optic illuminance detector is not shown in these views. 
centered in aluminum cylinders that are secured to the exterior of the box by aluminum rings that are glued in place to the exterior side of the box. Sufficient space surrounds the bulbs and their mounts to allow some air circulation to keep the interior of the box from overheating. A $2 \mathrm{~cm}$ diameter hole (not shown) is provided in the lid of the box above the upper bulb to help exhaust the heat. The reason for the internal heating is that the exit port is closed. The exit port is composed of a sandwich of a beveled plate to support additional structures and define the approximate size of the exit port, a polymethy methacrylate (PMMA) white transmissive diffuser approximately $3 \mathrm{~mm}$ thick, and a thin round circular mask defining the $15 \mathrm{~cm}$ exit port exterior. The reason the PMMA diffuser is needed is to support the fiber-optic illuminance head, not shown in Fig. 2. Additionally, the PMMA diffuser permits activity in the vicinity of the exit port without substantially perturbing the exit-port luminance and thereby improves the uniformity of the exit port luminance. It also prevents direct rays from the lamps to affect the results near the center of the exit port. The illuminance head pierces the PMMA diffuser at its center-the details of mounting the illuminance head are provided below.

LUMINANCE UNIFORMITY OF EXIT PORT: The uniformity of the exit port is measured using a luminance meter. Figure 3 shows the twelve measurement locations across the $15 \mathrm{~cm}$ exit port. The average source luminance $L_{\mathrm{s}}$ is $6228 \mathrm{~cd} / \mathrm{m}^{2}$ with a relative standard deviation of $0.7 \%$. During the course of the measurements, the average source photodiode current $J_{\mathrm{s}}$ is $8.108 \mu \mathrm{A}$, which provides a source luminance calibration of

$$
C_{\mathrm{s}}=L_{\mathrm{s}} / J_{\mathrm{s}}=768.2\left(\mathrm{~cd} / \mathrm{m}^{2}\right) / \mu \mathrm{A}
$$

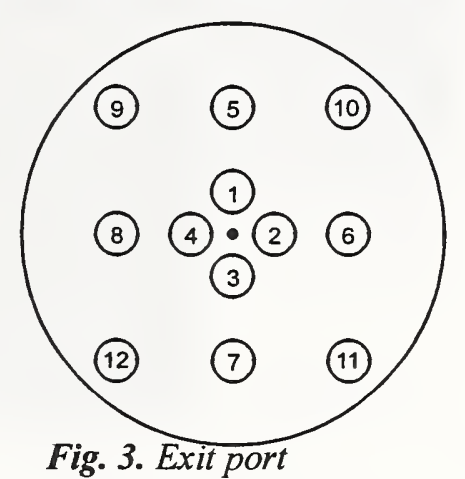

measurement locations.

to be based upon the photodiode source monitor current $J_{\mathrm{s}}$.

SOURCE STABILITY: The

luminance of the source as a function of time is measured between position $\# 8$ and \#4 at $30 \mathrm{~mm}$ to the left of center and is shown in Fig. 4.

Measurements of the luminance could not be made at the center because of the illuminance head located there. It is judged safe to begin taking any data after $60 \mathrm{~min}$ from the start of the lamps so that variations would be no more than approximately $1 \%$. The graph shows a very slow drift after approximately $40 \mathrm{~min}$. Usually the data collection time is much less than one hour; thus there is rarely any problem with source variations. The source photodiode current $J_{\mathrm{s}}$ is recorded for each data point collected so that any unexpected variation can be identified and possibly corrected. Given the uncertainties associated with these measurements, corrections from source variation are never required. The variations observed in the source luminance are partly due to the heating of the interior of the box. The output of the lamps is observed to change if the box is opened for even a brief period of time. The time required for reaching equilibrium after opening the box is on the order of $30 \mathrm{~min}$.

\section{SOURCE SPECTRUM: The} normalized radiance spectrum is shown in Fig. 5. The fluorescent lamps are listed as "daylight" bulbs. Fluorescent lamps are used in preference to tungsten halogen bulbs because of heating: The fact that we employ a closed exit port could create severe heating

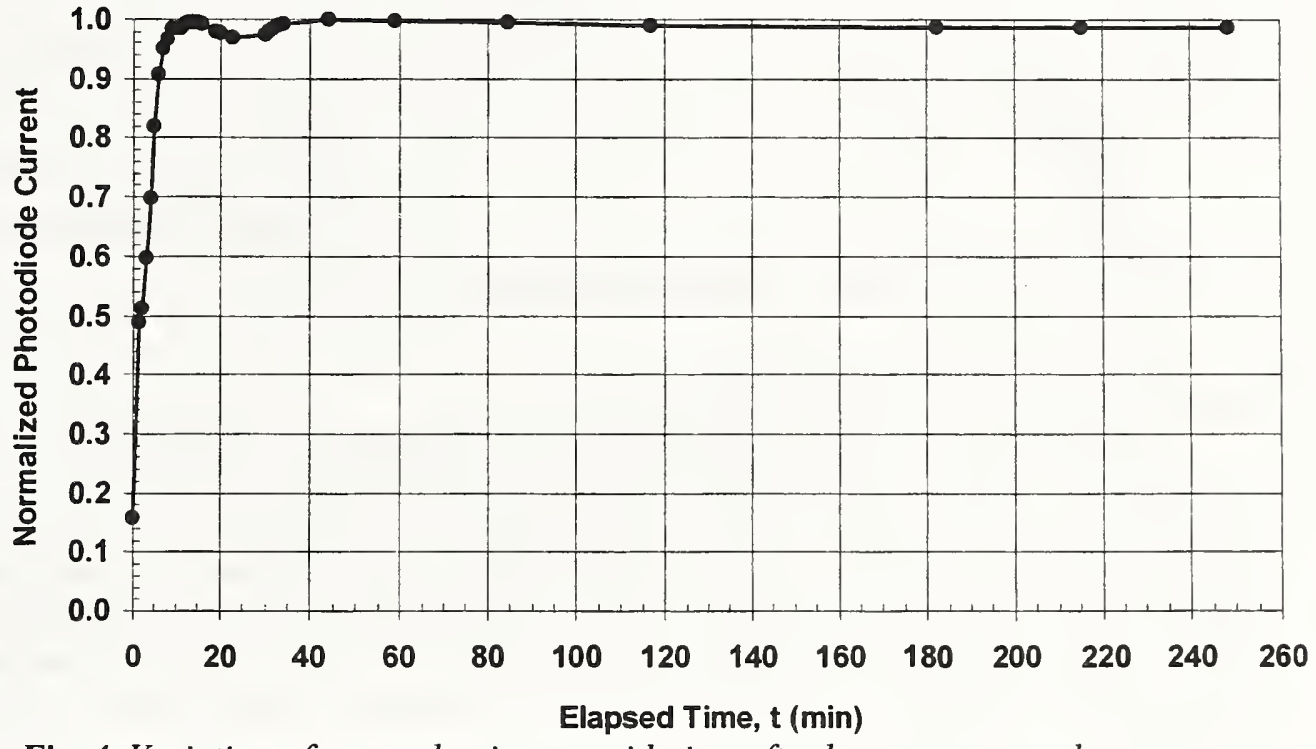

Fig. 4. Variation of source luminance with time after lamps are turned on.

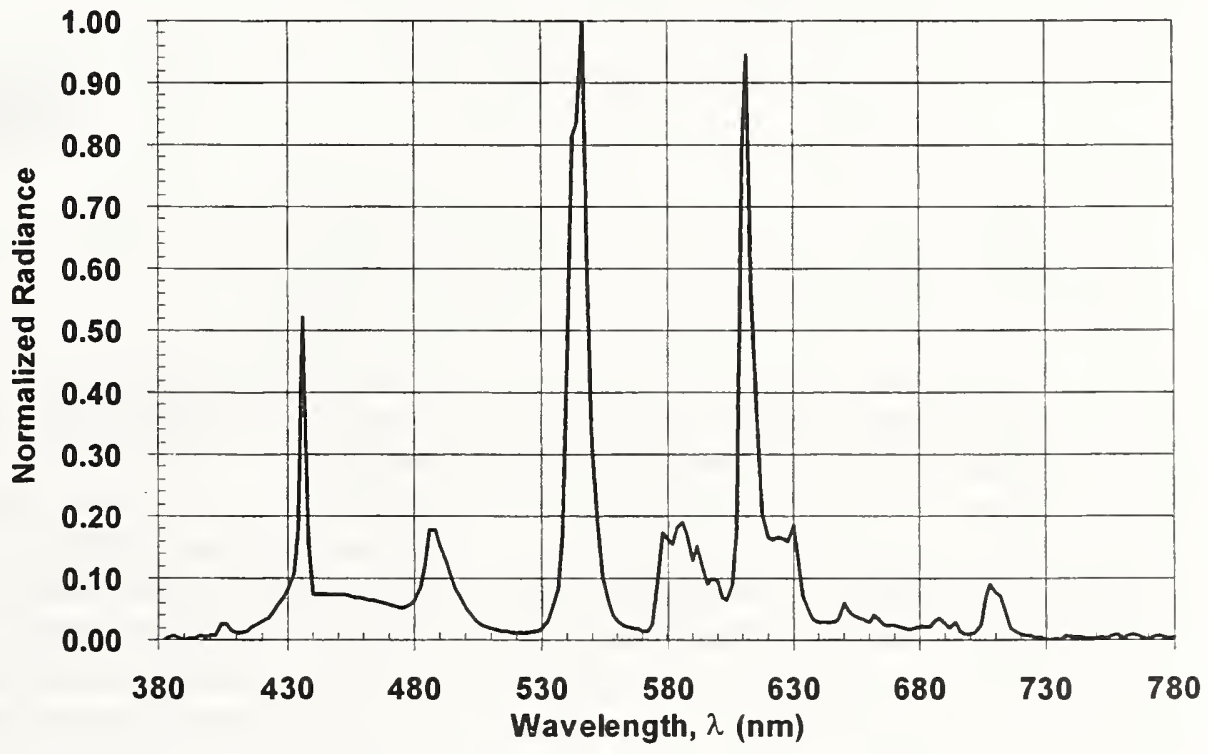

Fig. 5. Normalized source spectrum. 
problems if tungsten halogen bulbs were used. Ventilation fans might be used to offset such heating, but then there are complications from dust and dirt contaminating the interior of the box. No inconsistencies are observed that can be attributed to the source spectrum.

ANGULAR LUMINANCE DISTRIBUTION: The luminance of the source as a function of angle from the normal is measured between positions \#8 and $\# 4$ at $30 \mathrm{~mm}$ to the left of center and is shown in Fig. 6. A deviation of $5 \%$ from Lambertian behavior occurs at approximately $45^{\circ}$.
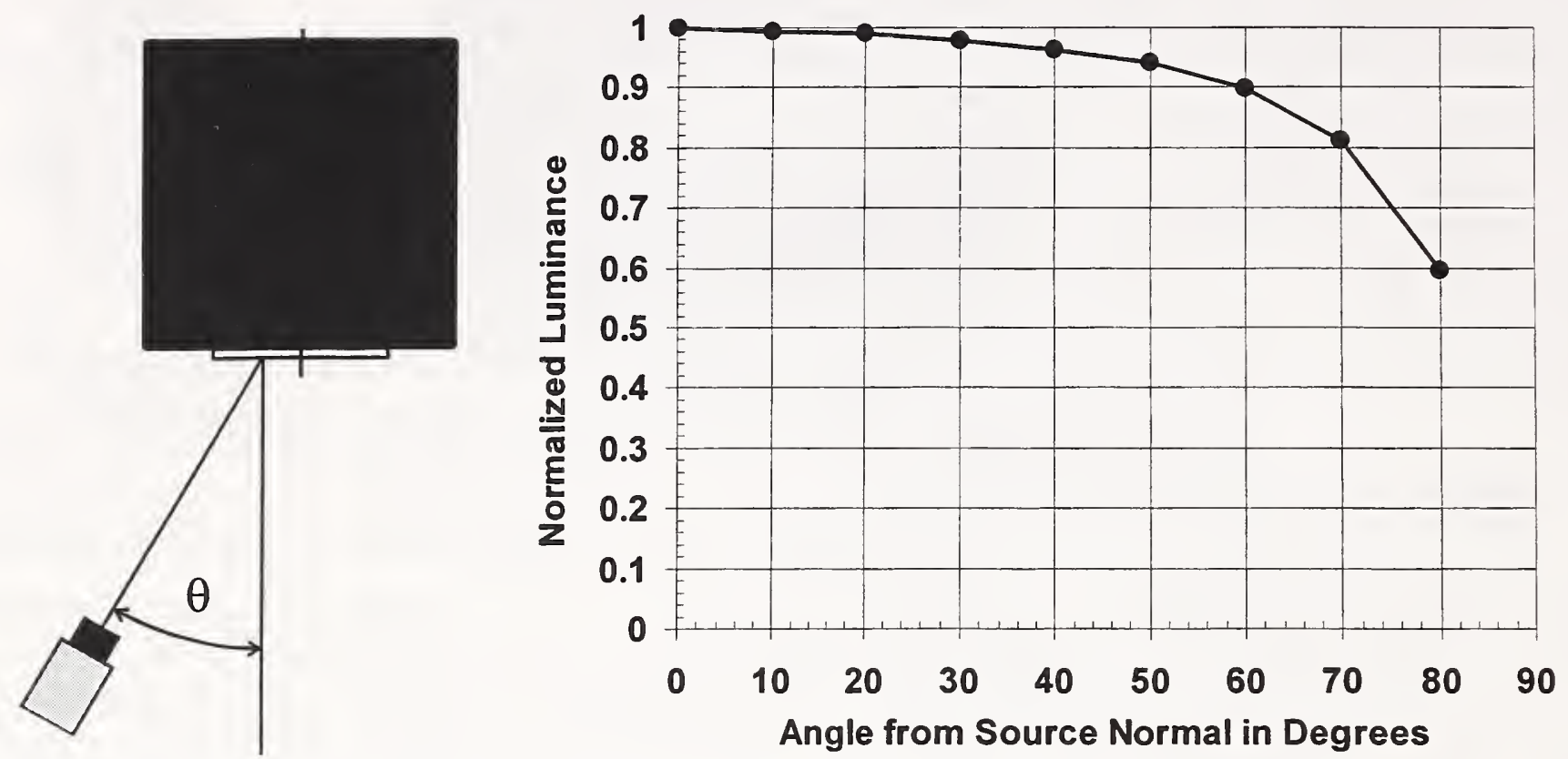

Fig. 6. Source luminance as a function of angle.

\section{ILLUMINANCE DETECTOR}

The main part of the illuminance detector is the illuminance head mounted at the center of the exit port of the integrating-box source. It serves as the input to the detector apparatus. The fiber-optic cable exits the box and enters a small integrating sphere to diffuse the output of the fiber optic and provide an input to the detector. The reason for such an integrating sphere is to integrate the light from the fiber optic and diffuse it. It also minimizes any geometric sensitivity to changes in how the light exits the fiber and enters the detector. The cost of such diffusion is a loss of signal. The fiber-optic cable is $3 \mathrm{~mm}$ in diameter, including the black sheath that surrounds a single $2 \mathrm{~mm}$ plastic fiber. Two types of detectors can be employed: (1) a photopic photodiode (PPD) and (2) a photopic photomultiplier tube (PMT). Each part will be explained separately.

FIBER-OPTIC ILLUMINANCE HEAD: The fiber-optic illuminance head is shown in cross-section in Fig. 7. (Approximately 60 different illuminance-head configurations were attempted before this design was chosen.) An aluminum rod is drilled out to $2.1 \mathrm{~mm}$ in diameter to readily accommodate the unjacketed $2 \mathrm{~mm}$ fiber without stressing the fiber. The other end is drilled out to $3 \mathrm{~mm}$ to provide a tight slide fit for the $3 \mathrm{~mm}$ jacketed fiber to avoid any light leaks from a lose fit. The bare fiber end is machined down to $3 \mathrm{~mm}$ to press fit into the $3 \mathrm{~mm}$ hole in the plastic diffuser covering the exit port of the source. The diffusion plug in the end of the illuminance head is made from a mixture of quick-hardening epoxy and reagent-grade

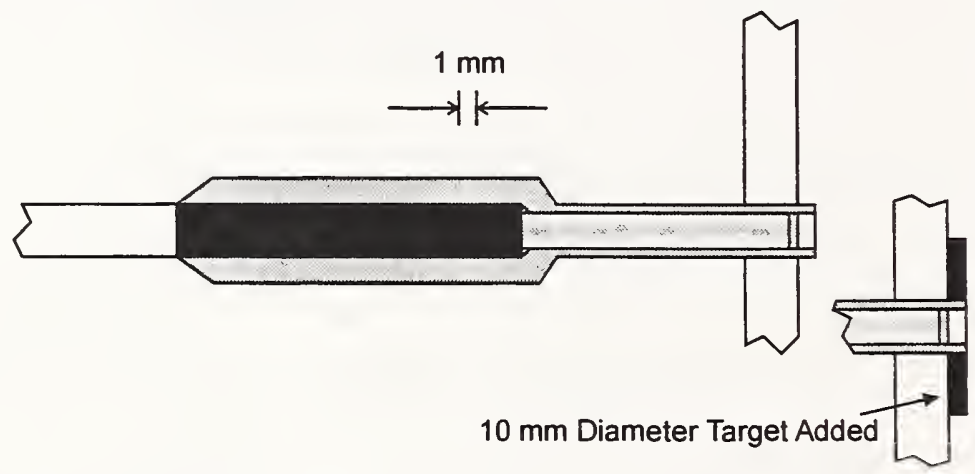

Fig. 7. Illuminance head cross section with and without $10 \mathrm{~mm}$ target. barium sulfate $\left(\mathrm{BaSO}_{4}\right)$. The mixture gets thick and needs to be mixed quite deliberately to eliminate any small clumps of the $\mathrm{BaSO}_{4}$ as much as possible. One end of a $1 \mathrm{~m}$ long fiber-optic cable is sanded with 600 -grit emery paper in a jig that holds the fiber perpendicularly to the emery paper. Some of the jacketing is removed from the cable so that when the fiber is inserted into the tube, the end of the fiber is just short of the end of the aluminum tube by approximately $1 \mathrm{~mm}$ or less. That end of the tube is then packed with the epoxy- $\mathrm{BaSO}_{4}$ mixture and allowed to harden. The fiber-optic cable is then withdrawn-the epoxy does stick to the surface of the fiber, but it is not hard to break the fiber away from the epoxy. The fiber is then re-sanded with 600 -grit emery paper tremove a small fraction of a millimeter of its length and re-inserted as shown in Fig. 7 . Because of the 
second sanding, the end of this fiber does not touch the interior side of the epoxy plug. The illuminance head with its cable is then press fit into the $3 \mathrm{~mm}$ hole in the exit-port diffuser so that it sticks out from the outside surface of the diffuser approximately $1 \mathrm{~mm}$. A $10 \mathrm{~mm}$ diameter disk target with a $3 \mathrm{~mm}$ central hole-an annulus-is added to provide a larger un-illuminated (dark) area to be measured by the probe. Figure 8 shows the final arrangement of the illuminance head surrounded by the $10 \mathrm{~mm}$ diameter annulus. The thickness of the annulus is adjusted so that the illuminance head sticks out slightly from the surface of the annulus. However, the annulus is thick enough to shield the illuminance head from any direct rays emanating from the exit port diffuser-such rays would seriously corrupt any luminance measurement attempted with the probe. The outside surface of the aluminum tube and the fiber-optic cable that resides within the box source are all painted with white latex paint to minimize any luminance perturbations near the exit port.

FIBER-OPTIC DIFFUSING SPHERE: The other end of the fiber-optic

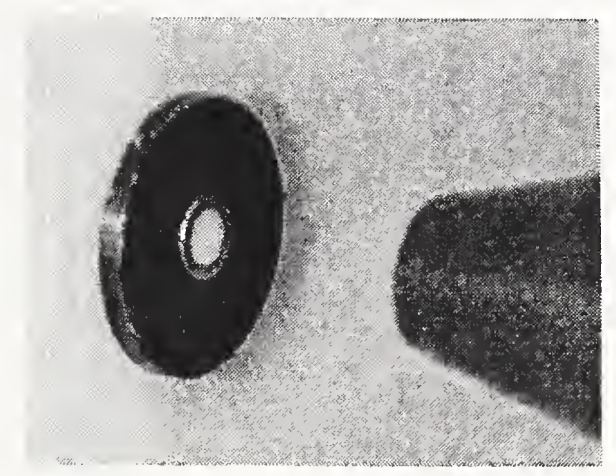

Fig. 8. Close-up of illuminance head with $10 \mathrm{~mm}$ target. Tip of a test probe is at the right.

cable (approximately $1 \mathrm{~m}$ long) that comes from the illuminance head is inserted into a small integrating sphere made from polytetrafluoroethylene (PTFE). (The PTFE is the normal kind available from plastic suppliers. It is not the high-reflectance kind. We wanted to use the high-reflectance kind of PTFE, but it was not available at the time of construction.) The sphere is machined in halves with the $8 \mathrm{~mm}$ hole for the detector made in one half. The two halves are machined and assembled into the mounting cube with virtually no clearance within the cube so they are easily press-fit into place. The $2 \mathrm{~mm}$ holes for the unjacketed fiber-optic cables are then drilled at the center of the side ports where the two halves press together. For this application, only one hole is used. The cube is mounted to an optical mounting post used in the laboratory via a short solid black acetal-plastic cylinder contained within a mounting tube attached to the bottom of the mounting cube-shown at the left in Fig. 10, where the mounting cube is shown attached to the PMT detector. The fiber-optic cable is fed through a black-acetal-plastic cylinder similarly mounted to one of the side ports. This fiber-optic mount has a $3 \mathrm{~mm}$ diameter center hole and is sealed with a small o-ring to prevent any light leaks into the sphere-see Fig. 10 left side, where the fiber-optic cable is seen projecting toward the bottom of the figure. The jacket of the fiber-optic cable is trimmed back so that the $2 \mathrm{~mm}$ end of the fiber extends almost all the way

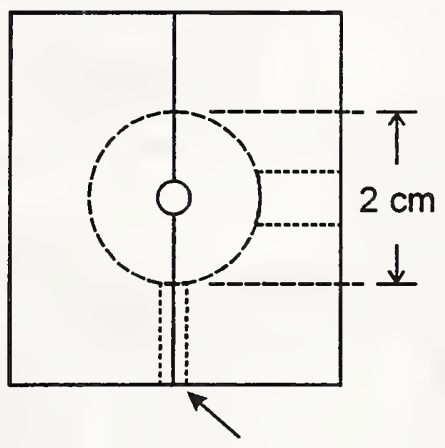

$2 \mathrm{~mm}$ Holes for Fiber Optic Cables

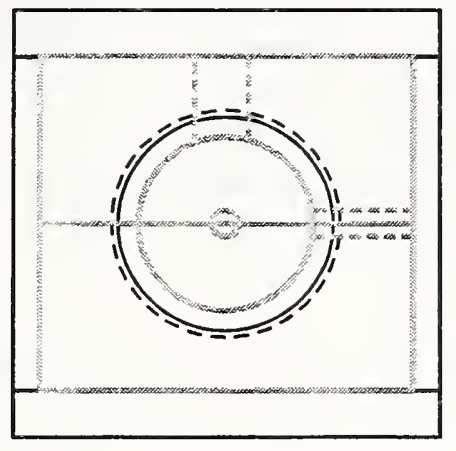

ThorLabs C4W 4-Way Mounting Cube with B2C Threaded Cover Plate on each end

Fig. 9. Fiber-optic diffusing sphere assembly shown with two fiber-optic input ports and one exit port for the detector. through the PTFE wall of the diffusing sphere.

PHOTOMULTIPLIER DETECTOR: The PMT is a commercially available module requiring only $15 \mathrm{~V}$ dc input. The module fits within the inside diameter of the black-acetal-plastic holder where (using a set screw) it is held up against a black rubber washer that presses against the photopic filter. A custom-made photopic filter is provided based upon the PMT manufacturer's spectral sensitivity information. The photopic response accuracy is estimated to be approximately within $5 \%$ of the spectral luminous efficiency curve, that is, $f_{1}^{\prime}=5 \%$. For all illumination conditions and settings for the PMT, a nonlinearity of less than $2 \%$ is observed in using the PMT for photopic measurements from the diffusing sphere. The nonlinearity is calculated according to CIE specifications for $f_{3}{ }^{9}$ In addition to the $15 \mathrm{~V}$ input, battery operation is provided for certain testing purposes, not for general use in these experiments. The circuitry is shown in Fig. 11. The ten-turn potentiometer offers a sensitivity adjustment range of from 0 to 1000 . The useful range of sensitivity settings, depending upon the light input, was from 200 to 900 -see Fig. 12. The probe-performance data reported below is taken with a setting of 600 (locked). The entire circuit including batteries is placed within a single chamber that screws onto the mounting cube as shown in Fig. 10. The entire apparatus is wrapped with black felt to prevent any possible light leaks. The PMT apparatus is the detector used to obtain the final measurement results reported in this document. 
Black Acetal Plastic

* Parts from ThorLabs

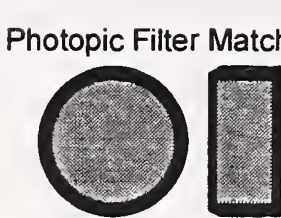

0.815 Diam. $\times 0.380$ thick.
PMT Module

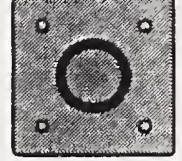

Hamamatsu H5783-01

$22 \mathrm{~mm}$ OD, $50 \mathrm{~mm}$ Long, $14 \mathrm{~mm}$ Bolt Grid, $10 \mathrm{~mm}$ Opening, $8 \mathrm{~mm}$ Effective Area

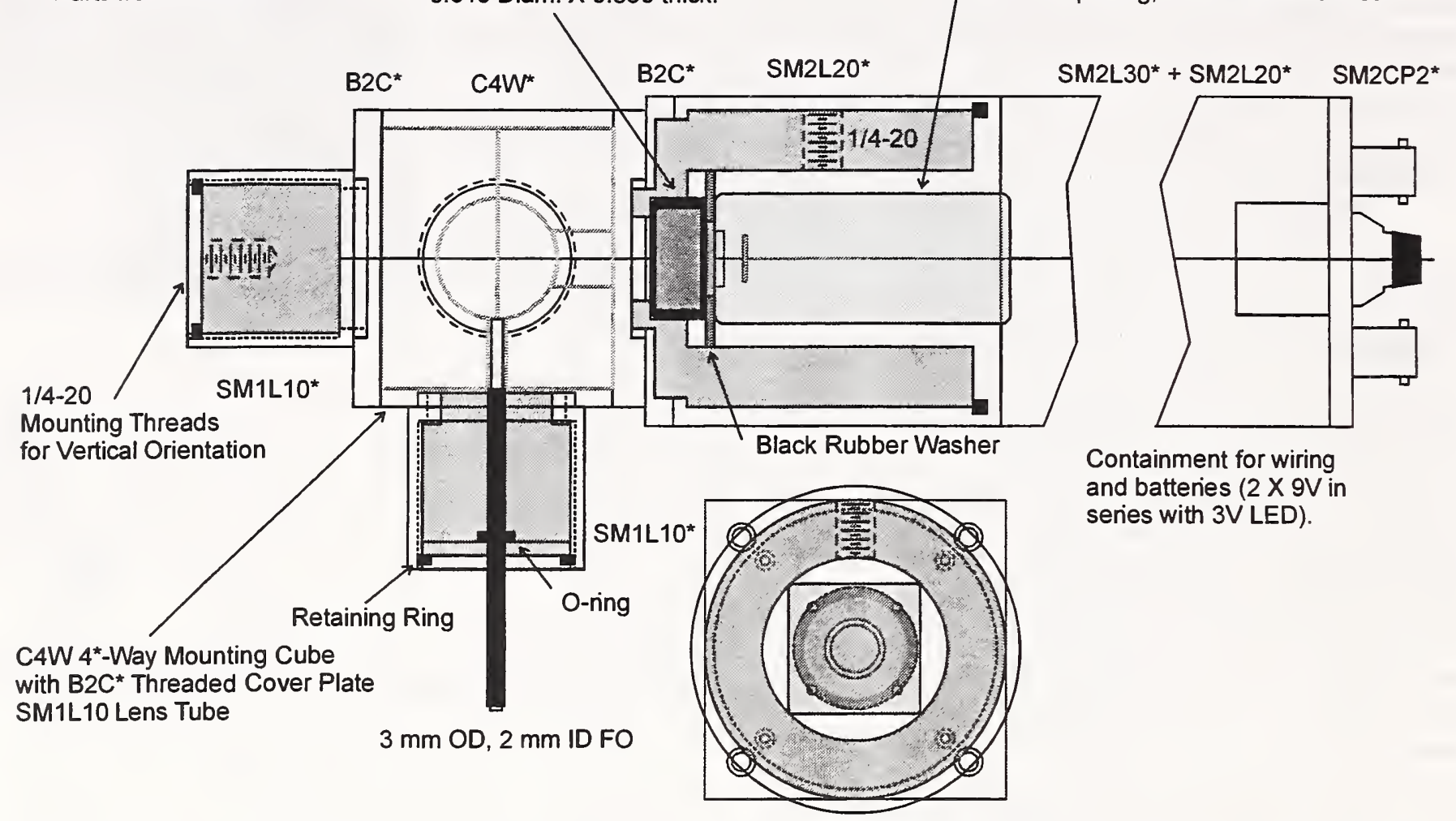

Fig. 10. Photopic photomultiplier assembly shown mounted to the diffusing sphere assembly. (Some measurements in inches, 1 in $=25.4 \mathrm{~mm}$.)

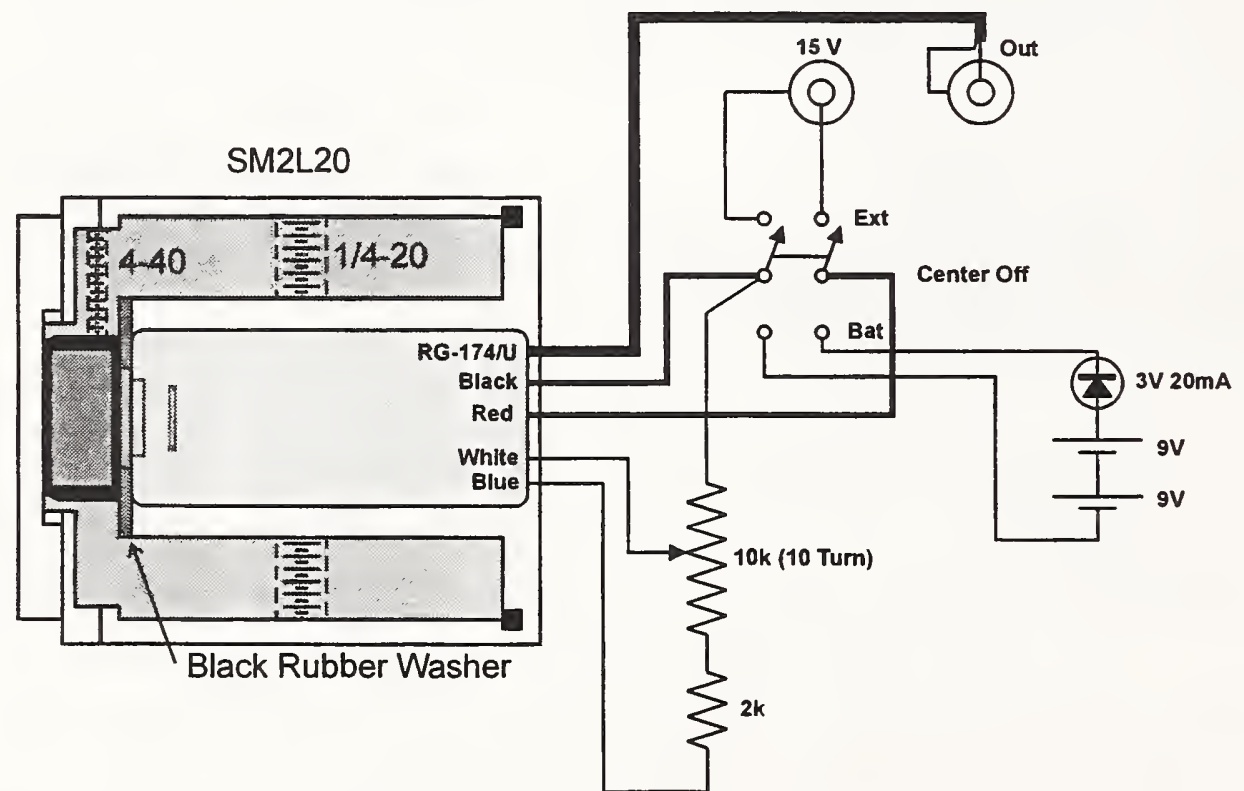

Fig. 11. Wiring diagram within the photomultiplier assembly. 


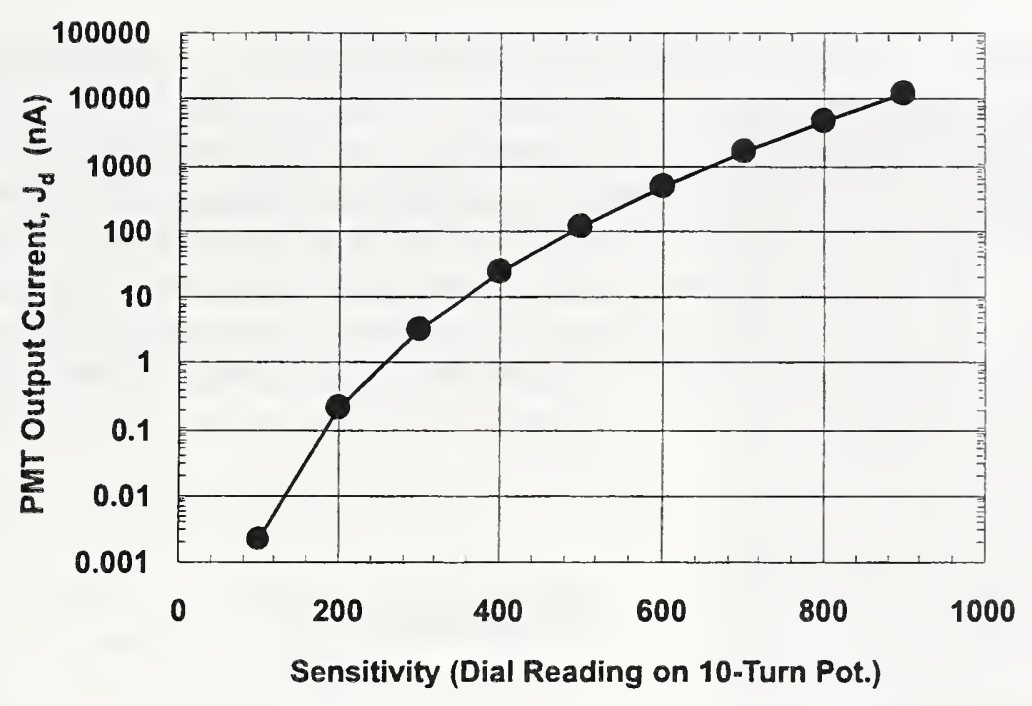

Fig. 12. PMT sensitivity vs. dial reading for an arbitrary illuminant.

PHOTODIODE DETECTOR: The photopic photodiode (PPD) can be used in place of the PMT detector; see Fig. 13. However, because of the insensitivity of the PPD as employed in this configuration, it is not used to obtain the final results for this document. It is included here for the sake of completeness. The employment of the PPD is limited to setup, testing, and alignment. For all illumination conditions the PPD is found to have a nonlinearity of less than $2 \%\left(f_{3}<2 \%{ }^{9}\right)$. The nonlinearity is considered to be primarily from the uncertainties in the current meter used to make the photodiode current measurements below $1 \mathrm{nA}$; such measurement results are complicated by a variety of noise and cabling problems over the distances used (approximately $5 \mathrm{~m}$ ).

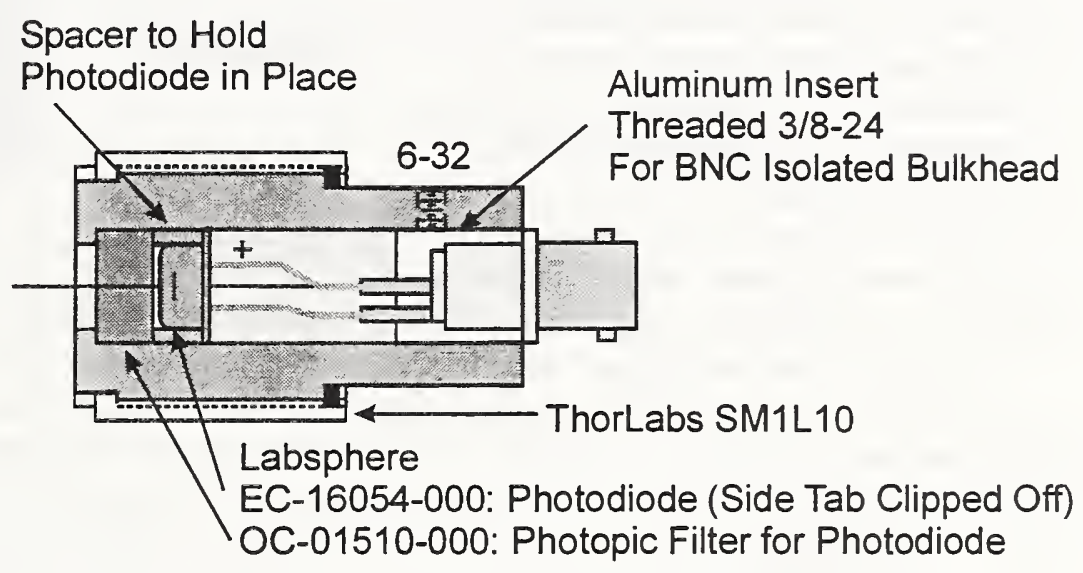

Fig. 13. Photopic photodiode detector assembly.
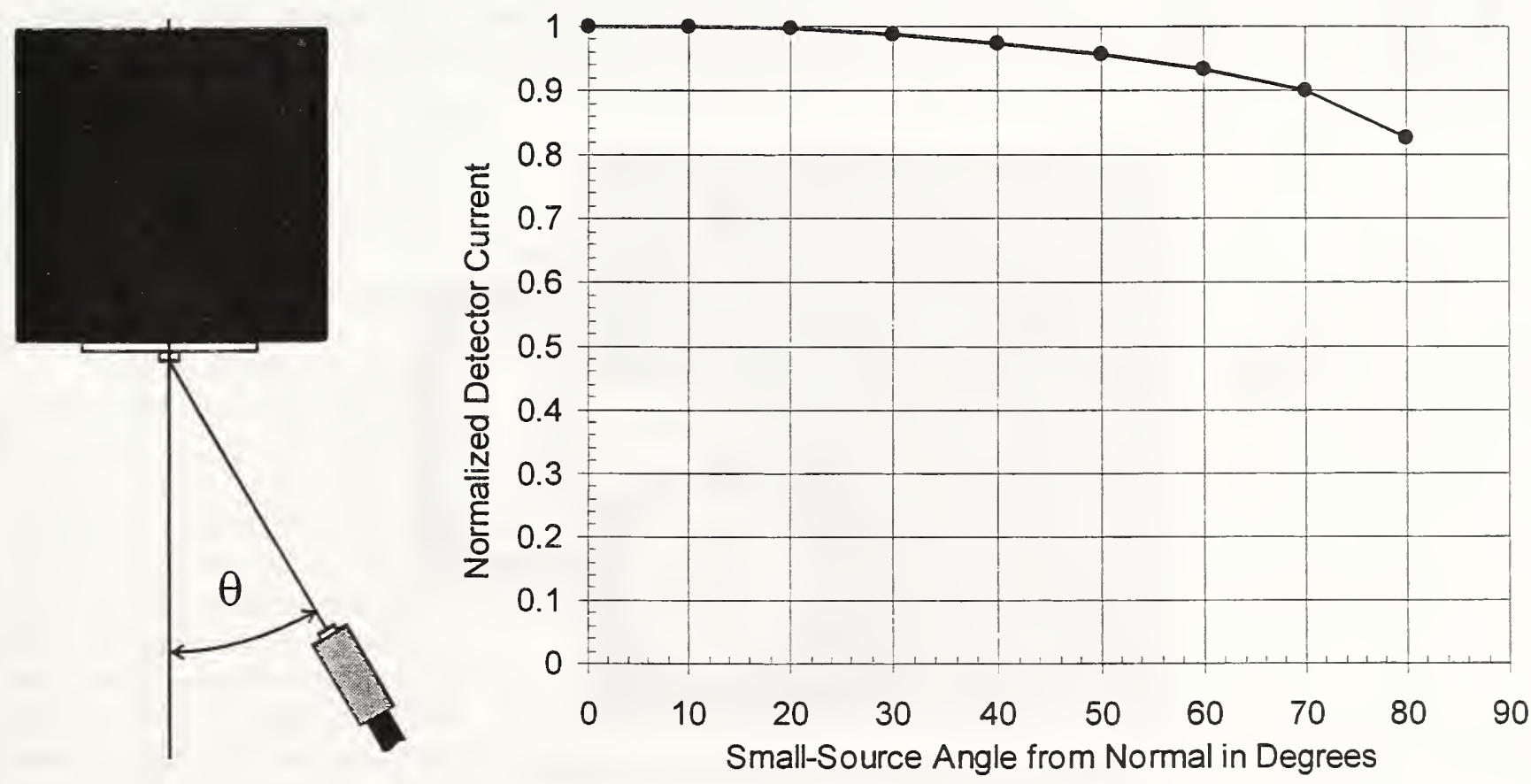

Fig. 14. Angular sensitivity of the illuminance head. 
ANGULAR RESPONSE: The angular response of the illuminance head is measured using a small fiber-optic light source placed $500 \mathrm{~mm}$ away from the illuminance head. The diameter of the fiber-optic bundle is $13 \mathrm{~mm}$, providing a subtense of $1.5^{\circ}$ from the perspective of the illuminance head. The measured response from the detector is adjusted according to $1 / \cos \theta$ to compensate for the increase of coverage area of the small source with angle. That is, since the source illuminance varies according to $E_{\mathrm{s}}=E_{0} \cos \theta$, where $E_{0}$ is the illuminance from the small source at the normal to the exit port, the uncorrected detector current $J_{\mathrm{s}}{ }^{\prime}$ using the small source must be corrected for uniform illuminance conditions via $J_{\mathrm{s}}=J_{\mathrm{s}}{ }^{\prime} / \cos \theta$. The results are shown in Fig. 14 where it is seen that only a $10 \%$ decrease in sensitivity is observed for large angles of $70^{\circ}$.

ILLUMINANCE HEAD CALIBRATION: The illuminance head is calibrated in two possible ways: using a small source as in determining the angular response just above, or using diffuse illumination. The PMT detector is calibrated at a dial setting of 600 .

Small Source: As with the angular-response measurement, a small fiber-optic source is placed $z_{\mathrm{s}}=500 \mathrm{~mm}$ away from the illuminance head-see Fig. 15. The PMT detector output current $J_{d}$ is measured. The light box with the illuminance head is moved approximately $15 \mathrm{~mm}$ back from the fiber-optic source so that a small calibrated illuminance head could be placed at the same position $z_{\mathrm{s}}$. The illuminance $E_{\mathrm{c}}$ of the small source is measured, and a small-source calibration constant is obtained:

$C_{\mathrm{d}}=E_{\mathrm{c}} / J_{\mathrm{d}}=0.252 \mathrm{~lx} / \mathrm{nA}$. It is this small source calibration constant that is used to

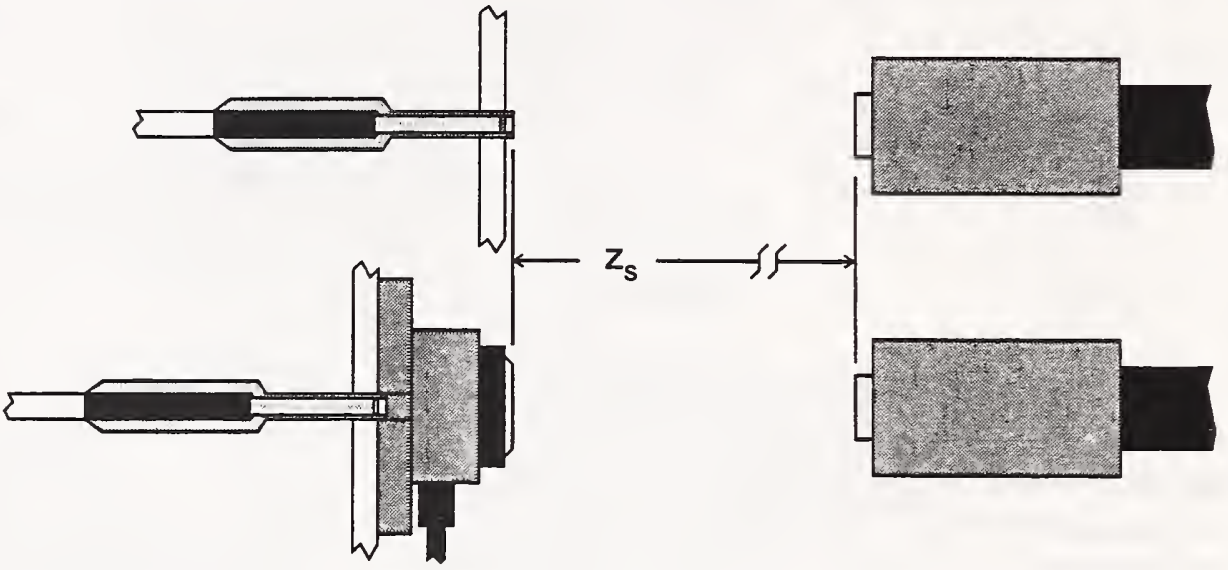

Fig. 15. Illuminance head calibration for small-source illumination. determine the illuminance arising from the backscattering from the measurement probe.

Diffuse Source: Figure 16 shows a side-view cross section of a $150 \mathrm{~mm}$ inner-diameter polystyrene-foam hemisphere that provides even diffuse illumination of the illuminance head. The polystyrene is white virgin closed-cell material of density $32 \mathrm{~kg} / \mathrm{m}^{3}$ (or $2 \mathrm{lb} / \mathrm{ft}^{3}$ ), approximately twice that used for common polystyrene-foam picnic coolers and house insulation (from which the box source is made). The illuminance $E_{\mathrm{h}}$ upon the wall of the hemisphere is measured by the same small calibrated illuminance head (not shown in Fig. 16) used with the small-source calibration whereby its measurement surface is placed parallel with the interior surface of the hemisphere at approximately $45^{\circ}$ from the normal of the box source exit port. The hemispherical calibration constant is obtained: $C_{\mathrm{h}}=E_{\mathrm{h}} / J_{\mathrm{d}}=0.265 \mathrm{~lx} / \mathrm{nA}$. After all the data are taken, this hemispherical calibration is repeated whereby it is found that $C_{\mathrm{h}}$ changes no more than $0.1 \%$. This calibration constant is not used for these data, however. The hemispherical calibration method is developed to provide quick checks of the performance of the apparatus during the course of the experiments. Checking the hemispherical calibration is important if the illuminance head acquires any dirt, dust, or if some change in the geometrical configuration within the detection system occurs during the course of the experiments.

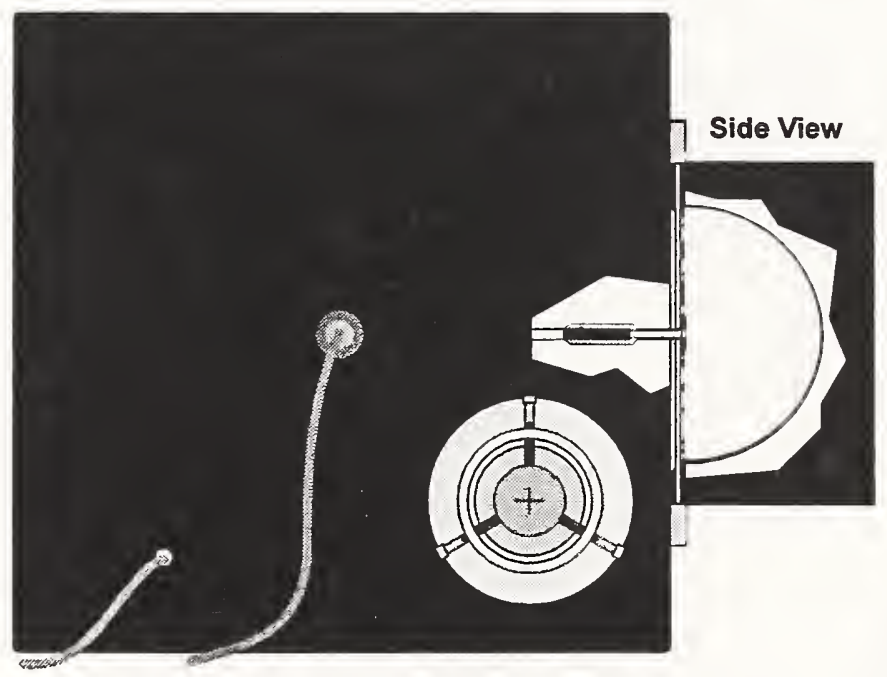

Fig. 16. Illuminance head calibration for diffuse illumination. 


\section{PROBE DESIGN}

The box source and probe apparatus is shown in Fig. 17. For careful alignment purposes, the probe's detector can be easily removed whereby a sighting hole in a black disk aligned with the normal of the boxsource exit port can be used to be sure that the probe is centered on the illuminance head. The probe is held in place by a black-plastic ring to provide a frictional slide fit. The mounting ring is attached to a long rail that is supported to avoid flexure of the rail. The rail assembly can be moved by a manual $x-y-z$-positioner. The entire assembly is placed upon a larger rail. Alignment of the probe with the rails is accomplished with a small He-Ne laser that defines the normal of the exit port of the box source. Alignment of the laser with the exit-port normal is within $0.5^{\circ}$. As mentioned previously, the box source is placed on a motorized $x-y-z-$ positioner that can be moved independently of the probe.

CONSTRUCTION: A cross section of the probe pointing at a display surface is shown in Fig. 18 along with the parameters that define the region of the surface that contributes to the measurement. The apex angle $\xi$ of the probe is $30^{\circ}$. The tip diameter of the narrow frustum is

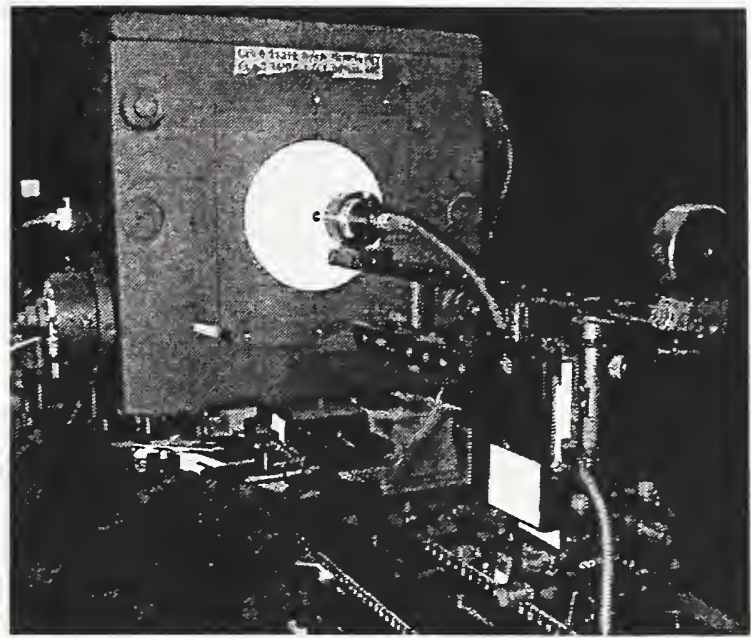

Fig. 17. Probe mounting apparatus and source. $d=5 \mathrm{~mm}$. The diameter of the cylindrical body is $38 \mathrm{~mm}$. There are three smaller internal frustums that are used to define the area under investigation and reduce the internal scattering within the probe. The internal frustums have apex angles of $90^{\circ}$. The purpose of the tip frustum is to minimize the amount of light that hits or passes through the first internal frustum. The second internal frustum has a larger tip diameter than either of the other internal frustums. Thus, the region of the source of light that can directly contribute to the measurement is defined by the diameters of the front and back internal frustums, $d_{1}$ and $d_{2}$, respectively. The fine solid lines from the detector to the display surface in the bottom part of the figure correspond to the dashed lines defined by arrows associated with the apertures $d_{1}$ and $d_{2}$ in the upper part of the figure. Provided that the detector is larger than the maximal rays defined by $w$, all the light coming from the region defined by $p$ can contribute to the measurement. This neglects any extraneous light that is scattered off the apertures or reflected within the probe through the internal frustums. All the interior of the probe is painted glossy black using high-quality enamel-as black as we could find. Except for scattering from the apertures of the internal frustums, the idea is to control the reflections of stray light by absorbing it with multiple reflections from the glossy surfaces. Thus, ideally, a ray that comes from outside the region defined by $p$ that enters through the front internal frustum will hit either the central or rear frustum and be multi-reflected away between the internal cylindrical wall and the frustum external surface. Determining how well the probe can control these internal reflections as well as how much light the probe scatters back onto the display surface to contaminate the measurement is the purpose of this work.

\section{SMALL FRUSTUM}

CREATION: The front frustum and internal frustums are vacuum formed from gloss-black vinyl-plastic disks having a thickness of $0.25 \mathrm{~mm}$ (10 mil). The vinyl disk is inserted into the holder and heated in place with a hot-air gun until the vinyl almost melts-as the vinyl heats up it goes from shiny and flat to slightly wrinkled, then to flat and dull, then to a very dull matte black just before it will become too soft to work well.
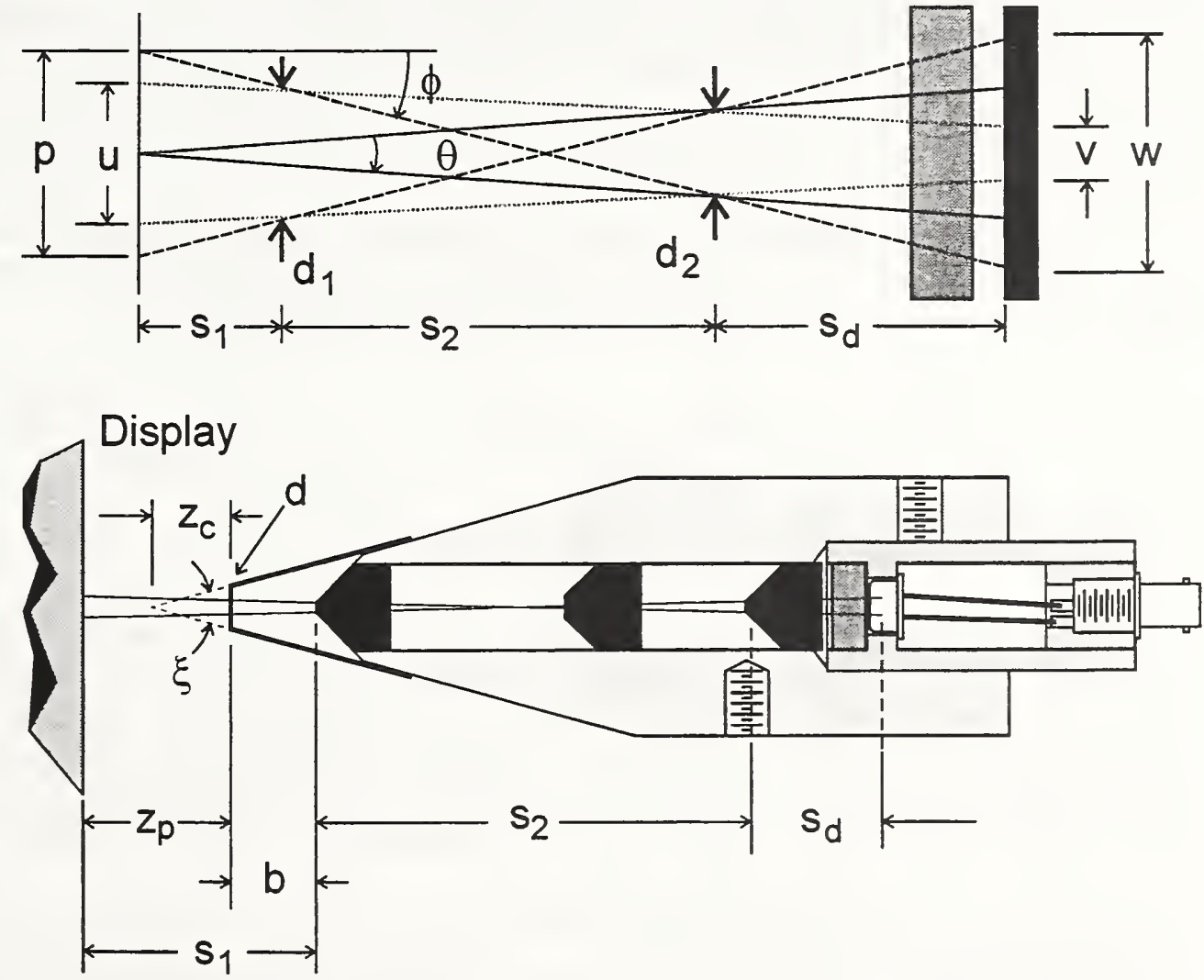

Fig. 18. Cross section of probe showing interior frusta and photopic photodiode probe detector and variables of interest. 

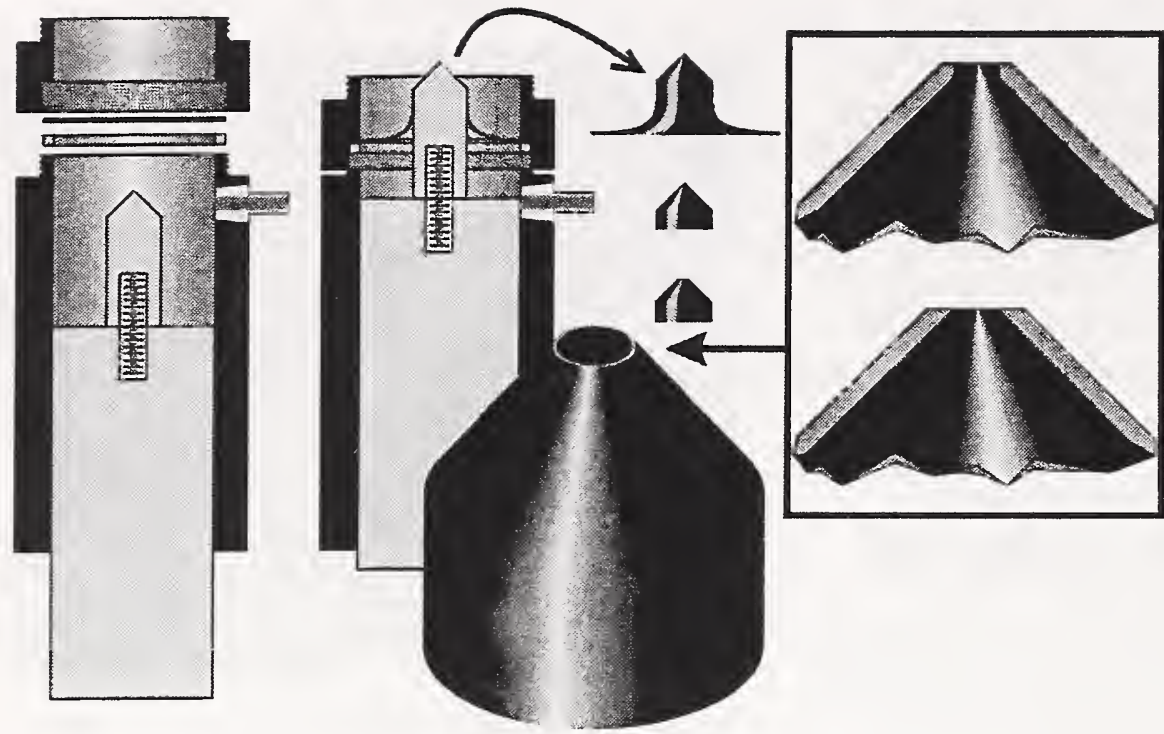

Fig. 19. Details of vacuum forming of small internal frusta.

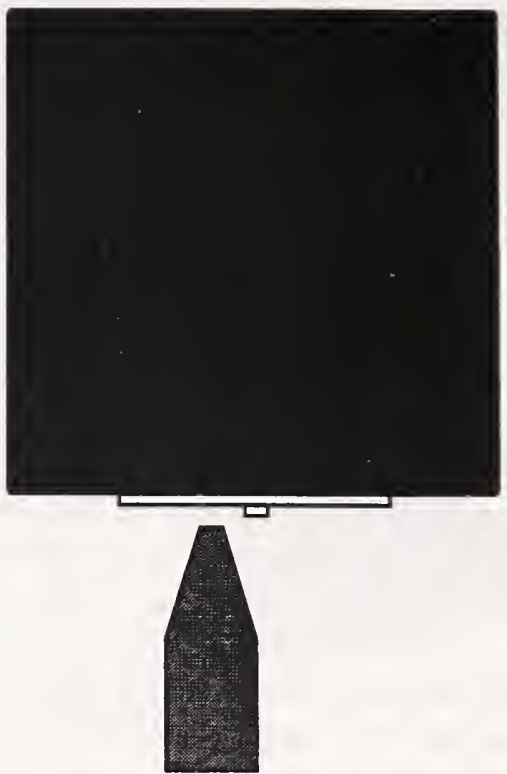

Fig. 20. Luminance calibration of probe.

Then a vacuum is applied that pushes the plunger into the disk so that the soft vinyl conforms to the shape of the plunger. Two shapes are used. One is a narrow cone to produce the main narrow frustum (the tip of the probe) with apex angle of $30^{\circ}$. The other shape is a cone with $90^{\circ}$ apex angle with cylindrical sides to fit within the body of the probe. The shaping of the apex hole of the frustum is performed under a low-magnification (20x) microscope using a small razor knife after it is gently drilled. Additionally, the apex of the frustum can be sanded with 600-grit emery paper so that the end is perpendicular to the frustum axis and the aperture edge is a sharp as possible. The frustum is cleaned with a blast of air and painted with gloss-black paint on the interior and exterior surfaces. In addition to assuring opacity of the now thinned material (on the order of $0.05 \mathrm{~mm}$ thick), the paint also serves to smooth out small imperfections in the edges of the shaped hole. For the configuration used in this report, the internal apertures are $1.6 \mathrm{~mm}, 3.2 \mathrm{~mm}$, and $1.6 \mathrm{~mm}$.

LUMINANCE CALIBRATION: The luminance $L_{\mathrm{s}}$ of the box source is measured a distance of $x=30 \mathrm{~mm}$ to the left of the illuminance head. The box source is then moved to the right a distance of $x=30$ mm so that the probe can measure the same region-see Fig. 20. The probe detector current $J_{\mathrm{d}}$ is obtained and a calibration constant is derived:

$C_{\mathrm{p}}=L_{\mathrm{s}} / J_{\mathrm{p}}=413\left(\mathrm{~cd} / \mathrm{m}^{2}\right) / \mathrm{nA}$. The probe luminance $L_{\mathrm{p}}$ based upon a probe detector current $J_{\mathrm{d}}$ is inferred to be given by $L_{\mathrm{p}}=C_{\mathrm{p}} J_{\mathrm{p}}$.

SENSITIVITY PROFILES: Using a slit source with $100 \mu \mathrm{m}$ width slits defined by two razor blades, the region of light that directly contributes to the probe response (the region defined by $p$ in Fig. 18) can be determined. Figure 21 shows the horizontal and vertical sensitivity profiles to have a width of approximately $3 \mathrm{~mm}$.
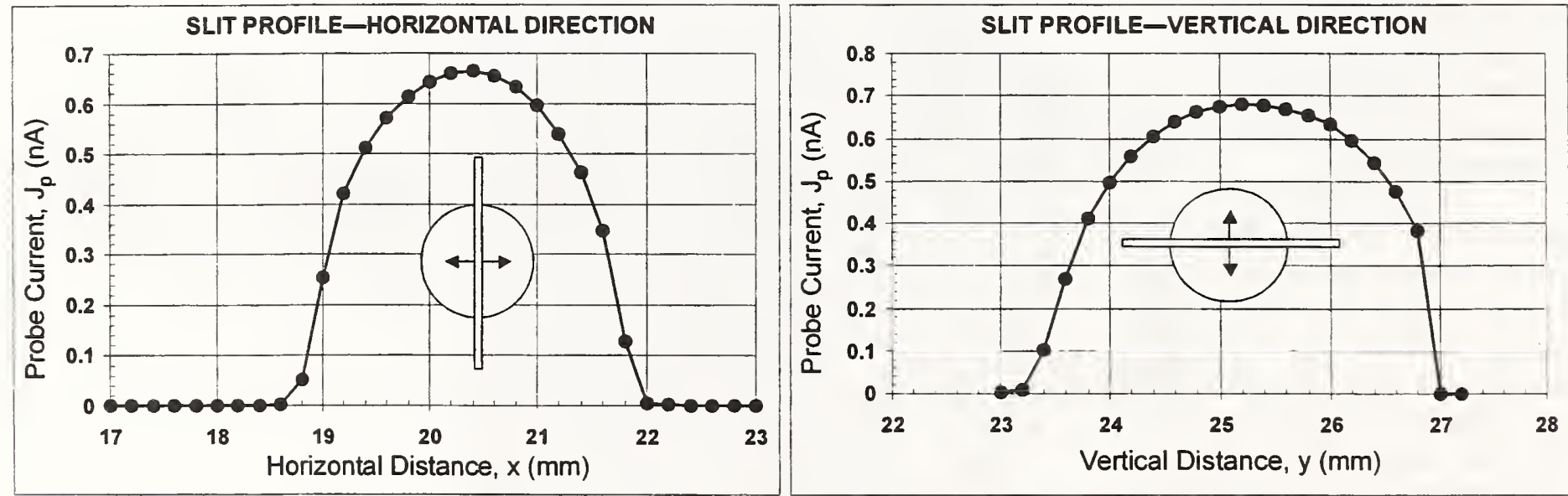

Fig. 21 Detection profiles of probe based upon $1 \mathrm{~mm}$ distance from nominally $100 \mu \mathrm{m}$ slits $\left(z_{\mathrm{p}}=1 \mathrm{~mm}\right)$. 


\section{RESULTS}

To see how well a probe design performs, we want to compare the inferred luminance $L_{\mathrm{p}}$ of the area under measurement and the illuminance $E_{\mathrm{p}}$ falling upon that area coming from back-scattering from the probe (and the rest of the apparatus) as the probe is moved back from the surface under measurement. Let $z_{\mathrm{p}}=0$ be the probe position where it just touches the surface of the annulus surrounding the illuminance head. If there are no light leaks of substance, both $L_{\mathrm{p}}$ and $E_{\mathrm{p}}$ should be essentially zero at $z_{\mathrm{p}}=0$, and they are for our purposes. The separation $z_{\mathrm{p}}$ of the probe from the measured surface is varied from $z_{\mathrm{p}}=0$ to $z_{\mathrm{p}}=50 \mathrm{~mm}$. In Fig. 22 we show the relative inferred luminance $L_{\mathrm{p}}(x)$ and relative illuminance $E_{\mathrm{p}}(x)$ based upon their maximum values obtained over the interval $0 \leq z_{\mathrm{p}} \leq 50 \mathrm{~mm}$. The luminance $L_{\mathrm{p}}$ that would be inferred from the photodiode current $J_{\mathrm{p}}$ bears little or no relationship with the reflected illuminance $E_{\mathrm{p}}$ falling back upon the target area because of reflection from the probe and the apparatus. The illuminance $E_{\mathrm{p}}$ peaks near $z_{\mathrm{p}}=10 \mathrm{~mm}$ whereas the inferred luminance $L_{\mathrm{p}}$ monotonically increases. These results indicate that the principal contribution to the measured luminance is the light scattering within the probe rather than contamination of the luminance resulting from backscattering from the probe onto the target area. For such a probe, if the target were absolutely black (infinite contrast), a measurement using the probe would indicate a contrast of 1000:1 at a distance of $z_{\mathrm{p}}=5.7 \mathrm{~mm}$ as indicated on the graph. Increasing the distance further would render lower contrasts.

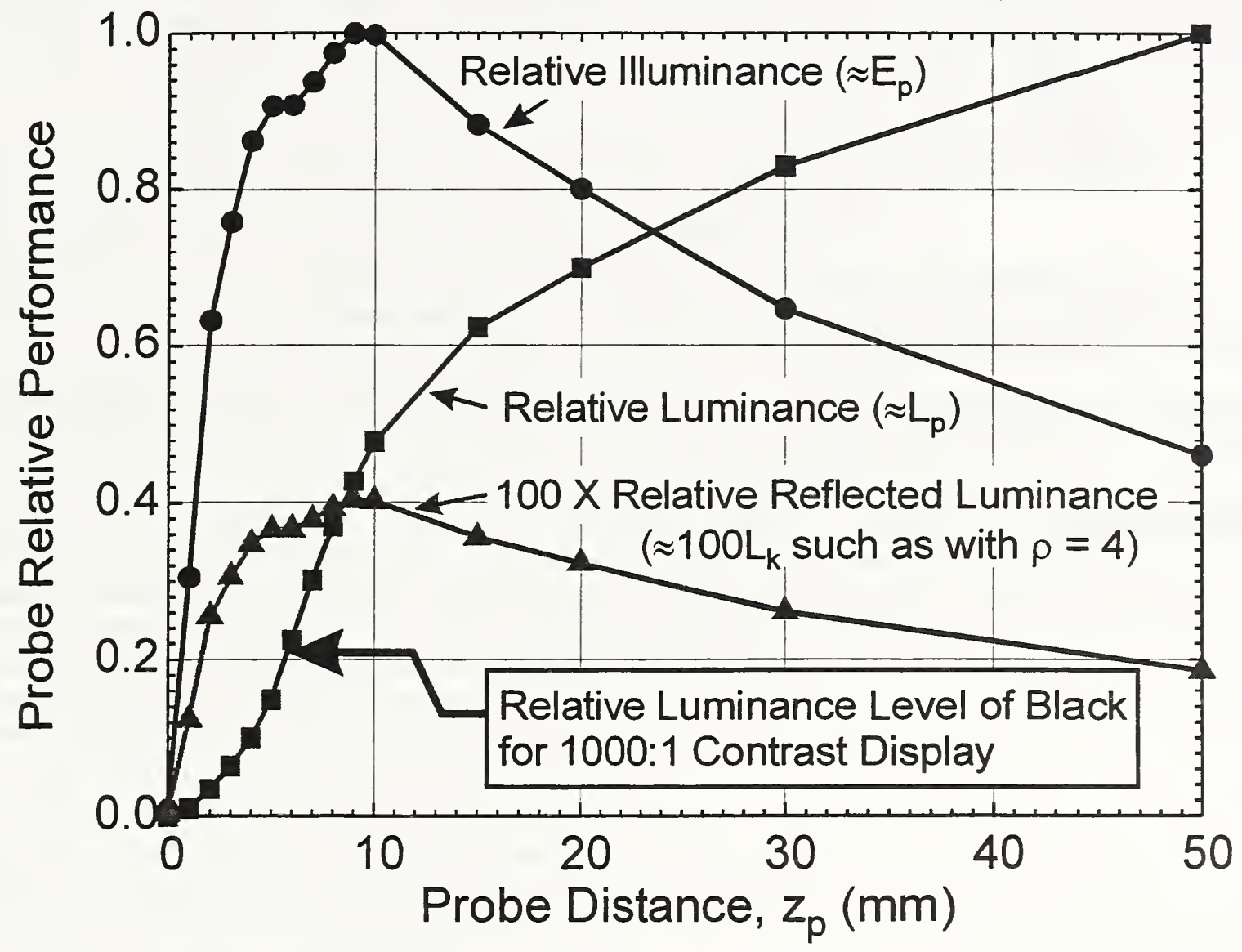

Fig. 22. Relative performance of probe as a function of the distance from the illuminance head.

To see just how serious the internal scattering is, we can imagine placing a black matte disk of plastic $10 \mathrm{~mm}$ in diameter with diffuse reflectance of $\rho_{\mathrm{K}}=0.040$ at the location of the target and calculate the luminance $L_{\mathrm{K}}$ that would be created from the backscattered illuminance $E_{\mathrm{p}}$ from the probe: $L_{\mathrm{K}}=\rho_{\mathrm{K}} E_{\mathrm{p}} / \pi$. The plot of $L_{\mathrm{K}}$ would have the same shape as the illuminance $E_{\mathrm{p}}$, but on the scale of the graph in Fig. 22, $L_{\mathrm{K}}\left(z_{\mathrm{p}}\right)$ it is too small to be appreciated. Multiplying $L_{\mathrm{K}}$ by 100 $\left[100 \times L_{\mathrm{K}}\left(z_{\mathrm{p}}\right)\right]$ renders it visible. Clearly, our main problem with this probe design is stray light within the probe itself and not backscattering of light from the probe apparatus back on the subject being measured. This fact is somewhat of a disappointment.

In Fig. 23 we compare the inferred luminance $L\left(z_{\mathrm{p}}\right)$ of the illuminance head with the inferred luminance $L_{\mathrm{W}}\left(z_{\mathrm{p}}\right)$ of a white matte plastic target of $10 \mathrm{~mm}$ in diameter having a diffuse reflectance of $\rho_{\mathrm{W}}=0.88$, and the inferred luminance $L_{\mathrm{K}}\left(z_{\mathrm{p}}\right)$ of a black matte plastic target $10 \mathrm{~mm}$ in diameter having a diffuse reflectance of $\rho_{K}=0.04$. The fact that the results obtained using the white target aren't some twenty times the results from the black target at nontrivial distances underscores that the 


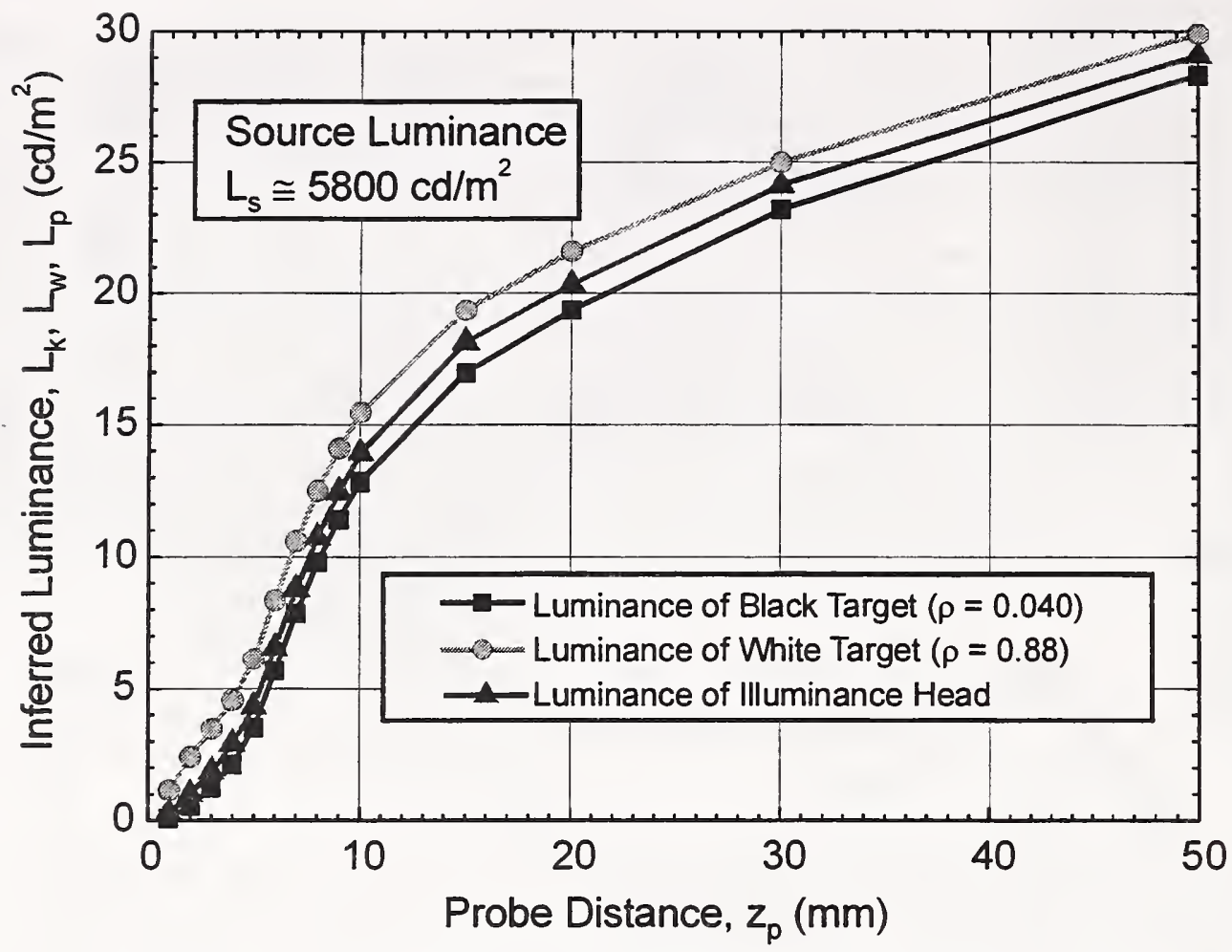

Fig. 23. Comparison of probe inferred luminance readings for a black

$10 \mathrm{~mm}$ target, a white $10 \mathrm{~mm}$ target, and the illuminance head with

$10 \mathrm{~mm}$ annular black target.

corruption to the luminance measurement is internal to the probe. The result for a sample of $x$-ray film would fall somewhere between the results from the white and black targets.

\section{CONCLUSION AND FUTURE DIRECTIONS}

It has been assumed that the use of these kinds of probes greatly improved the accuracy of measurements of small areas in displayed information over other methods, such as the use of a digital camera. This may well still be true when the probe is close to the surface being measured, that is, for small distances from the target. However, as the distance increases, the performance capability of the probe diminishes considerably owing to internal scattering from the probe. One type of scattering cannot be eliminated: The scattering off the edges of the apertures will persist no matter what we do. As the apertures get smaller, the edge-scattered light becomes more important as a contaminant. Consider the following: The light from the measured area goes as $\pi r^{2}$, where $r$ is the radius of the aperture. The contamination from edge scattering goes as the circumference $2 \pi r$. The measured light is hoped to be proportional to the area observed but is actually the sum of these two components. Because we assume the area measurement to be the desired result, we will extract it from the sum of the two contributions: $\pi r^{2}(1+2 / r)$. The second term is the contribution from the edge. Notice how as $r$ gets small the circumferential edge term gets large. We have left out any relative contribution constant from this equation. It is only to illustrate the problem, not to predict the result.

The other problem is stray light reflection within the probe (not from edge scattering). It was hoped that with a glossblack interior, better control of these reflections would be achieved. Perhaps so, but it is yet insufficient to completely control the internal scattering. Perhaps better baffling with a more judicious choice of internal frustum placement will improve matters. However, there may be a solution that will virtually eliminate all these problems.

The current probe construction employs a photodiode. The photodiode does not discriminate the origin of the light. Whatever light hits it, it will measure. We have previously used a charge-coupled-device (CCD) camera with such probes, and have obtained good results. ${ }^{6}$ It would be valuable to repeat the analysis contained here using a detector that employed a lens to discriminate the light it would measure, such as a CCD camera. Hopefully, this will be the next step in attempting to make accurate light measurements of small areas of high-contrast images.

\section{ACKNOWLEDGEMENT}

We would like to express our thanks to the Advanced Technology Program (ATP) of NIST and to Elissa I. Sobolewski, Program Manager, Electronics and Photonics Technology Office of the ATP. This entire project was funded by the ATP. 


\section{REFERENCES}

${ }^{1}$ E. F. Kelley, "Fundamentals of Display Metrology," 2002 Society for Information Display International Symposium Short Course S-3, 2002 SID International Symposium, Boston, Massachusetts, 205 pp., May 19-24, 2002.

(ftp://ftp.fpdl.nist.gov/pub/seminars/SID02ShortCourseFunDM.pdf)

${ }^{2}$ CIE Publication No. 69, Methods of Characterizing Illuminance and Luminance Meters, Section 3.4.2.4 L "Measurement of the effect of the surrounding field." pp. 16-17.

${ }^{3}$ P. A. Boynton and E. F. Kelley, "Measuring the Contrast Ratio of Displays: It's Not Trivial," Information Display, Vol. 12, No. 11, pp. 24-27, November 1996. See also P. A. Boynton and E. F. Kelley, "Accurate Contrast-Ratio Measurements Using a Cone Mask," 1997 Society for Information Display (SID) Symposium, Digest of Technical Papers, May 11-16, 1997, Boston, MA, Sec 32.1, pp. 823-826 (May 1997). (ftp://ftp.fpdl.nist.gov/pub/straylight/ConeConSID97.PDF)

${ }^{4}$ P. A. Boynton and E. F. Kelley, "Compensation for Stray Light in Projection-Display Metrology," 2001-SID International Symposium Digest of Technical Papers, Society for Information Display, Vol. 32, pp. 334-337, San Jose, CA, June 4-8, 2001. (ftp://ftp.fpdl.nist.gov/pub/projection/SLET 03.pdf). Also see P. A. Boynton and E. F. Kelley, "NIST Stray Light Elimination Tube Prototype," NISTIR 6881, February 2002.( ftp://ftp.fpdl.nist.gov/pub/projection/SLET 04.pdf)

${ }^{5}$ A. Badano and M. J. Flynn, "Method for measuring veiling glare in high performance display devices," Applied Optics, Vol. 39, No. 13, pp. 2059-2066, May 2000. The original disclosure is found in: A. Badano, M. J. Flynn, and J. Kanicki, "Small-Spot Contrast Measurements in High-Performance Displays," Digest of Technical Papers, 1999 Society for Information Display International Symposium, San Jose, CA, Vol. 30, pp. 516-519, May 18-20, 1999.

${ }^{6}$ J. W. Roberts and E. F. Kelley, "Measurements of Static Noise in Display Images," Proceedings of the SPIE V4295B-27, Electronic Imaging Symposium, San Jose, CA, pp. 211-218, January 23, 2001. (ftp://ftp.fpdl.nist.gov/pub/straylight/SPIE01-StaticNoiseCCD-Meas.pdf)

${ }^{7}$ Certain commercial equipment, instruments, materials, systems, and trade names are identified in this paper in order to specify or identify technologies adequately. Such identification is not intended to imply recommendation or endorsement by the National Institute of Standards and Technology, nor is it intended to imply that the systems or products identified are necessarily the best available for the purpose.

${ }^{8}$ E. F. Kelley, "Polystyrene Box Uniform Light Sources," Council for Optical Radiation Measurements, May 4-6, 1999. (ftp://ftp.fpdl.nist.gov/pub/sources/PolystyreneBoxSourceCORM99.PDF)

${ }^{9}$ CIE Publication No. 69, loc. cit., pp. 20-21. Note that the equation and description presented in this manual have some typographical errors. The quantity $f_{3}(Y)$ and $f_{3}$ [the maximum of $f_{3}(Y)$ for the range of the instrument used] are actually measures of nonlinearity—not linearity. Equation (38) should read: $f_{3}(Y)=\left(\frac{Y}{Y_{\text {limit }}} \frac{X_{\text {limit }}}{X}-1\right) 100 \%$, where $Y$ is the instrument reading and $X$ is the source reading and the limit refers to the largest in the range employed. 

